\title{
Broken Time Reversal-Symmetry State in a Long Josephson Junction
}

\author{
Bal Ram Ghimire \\ Central Department of Physics \\ Tribhuvan University, Kirtipur, Kathmandu \\ E-mail:- balramghimire@gmail.com
}

\begin{abstract}
The possibility of a broken time-reversal symmetry state (BTRS) of the ground state of a single Long Josephson Junction (LJJ) due to the presence of additional tunneling channels is investigated by using a microscopic model for two-gap superconductors. The consequence of this broken time reversal ground state is discussed.The ground state phase configuration was obtained by minimizing the free energy of the LJJ with respect to phase variables in the absence of an external magnetic field. The boundary conditions were obtained at the junction interface. Applying the boundary conditions, the conditions for time-reversal symmetry invariance (TRSI) state and BTRS state were obtained. The relative phase in the ground state was numerically calculated. The phase configuration of the ground state was determined from the free energy calculation as a functions of relative phases $\left(\chi_{1}, \chi_{2}\right)$ for different values of inter-band current and Josephson current. When the ground state corresponds to the BTRS, the relative phase constant $\delta \theta^{\circ}$ computed as a function of phase difference $\phi^{\text {sS }}$ showed linearly dependence. The variation of current density $\left\lfloor J_{1}^{S}\right\rfloor$ with the phase difference $\phi^{\text {ss }}$ behaves as the sine-curve.
\end{abstract}

Keywords: Superconductor, Josephson interaction, Ohmic quasi-particle, biquadratic interaction, magnetic field.

\section{INTRODUCTION}

Due to relative phase of the two condensates of two-gap superconductor in a LJJ an interesting phenomenon in the ground state of the system may be produced. One of these interesting properties is a broken time reversal symmetry state. In the absence of external magnetic field, there are no net currents in the ground state of a LJJ. However, in the LJJ with one-gap and two-gap superconductor layer, the ground state satisfies the condition of no net current density via maintaining the inter-band phase difference of either 0 or $\pi$. This state is called the time-reversal symmetry invariant (TRSI) state. On the other hand, if the phase difference between the two condensates differs from 0 or $\pi$, then system is said to have phase frustration. When the phase frustration is maintained, the ground state may have non-zero current density in the absence of an external magnetic field while satisfying the condition of zero net current flow. This state of the ground state is called the broken time-reversal symmetry state (BTRS). Theoretical studies of superconductor-insulatorsuperconductor (hetero-Josephson) junctions between one- and two-gap superconductors suggest that the timereversal symmetry is violated in the ground state.

The relative phases of the condensates in the ground state reflect the time-reversal symmetry of the junction.
To study the TRSI and BTRS state, one needs to focus on the phase frustration. Phase frustration may also occur in a Josephson junction. This situation is similar to a two-gap superconductor with both inter-band Josephson and biquadratic interaction. A junction between a two-gap superconductor with either the $S_{++}$ or $S_{+-}$-symmetry and a conventional S-wave superconductor may yield a different behavior due to either absence or presence of phase frustration, respectively. The time-reversal symmetry breaking within two-gap superconductors was studied by Lee $e t$ al. (2009), Lin and Hu (2011), Tanaka et al. (2010) 2023-2026, Tanaka et al. (2010) 1010-1012 and Lin and $\mathrm{Hu}$ (2011). They realized that the inter-band Josephson interaction tends to lock the relative phase to either 0 or $\pi$, while the biquadratic interaction tends to lock the relative phase to $\pm \pi / 2$. Tanaka and coworkers claim that when the inter-band coupling $J$ is greater than zero (i.e. $J>0$ ), the Josephson interaction in the $S_{+_{+}}$ symmetry state will lock the relative phase $\chi$ to 0 . However, when $J<0$, the Josephson interaction in the $S_{+-}$-symmetry state will lock the relative phase to $\pi$. If the phase difference between two condensates differs from the phase-locked value of either 0 or $\pi$, then there is phase frustration which breaks the time-reversal symmetry in the ground state. 
The phase frustration and the BTRS state can also be described in terms of boundary conditions in the junction interface. Boundary conditions in the junction interface are described in terms of current density of the individual condensate. When the non-zero individual currents satisfy the condition of zero net current flow in the ground state, the system is strongly frustrated, resulting in broken time-reversal symmetry state. In this work the time reversal symmetry invariant and broken time-reversal symmetry state in junction with two-gap superconductors will be investigated.

\section{Possibility of Phase Frustration}

The possibility of phase frustration in a two-gap superconductor is reviewed by computing the free energy of the system. Earlier studies (Tanaka et al. 2010, Lin \& Hu 2011, Chung et al. 2002, Bardeen et al. 1957, Loufwander et al. 2000, Garaud et al. 2011, Hu \& Wang 2011, Platt et al. 2011) indicate that phase frustration and the appearance of broken time reversal symmetry state in a tunnel junction between two-gap and one-gap superconductors are closely related.

The ground state of the junction may be examined by computing the free energy. The free energy for the twogap superconductor in the absence of an external magnetic field is given by

$F=\alpha_{s}\left|\psi_{l}^{s}\right|^{2}+\widetilde{K}_{s}\left(\nabla \theta^{s}\right)^{2}+\beta_{s}\left|\psi_{l}^{s}\right|^{4}+\alpha_{d}\left|\psi_{l}^{d}\right|^{2}+$

$\widetilde{K}_{d}\left(\nabla \theta^{d}\right)^{2}+\beta_{d}\left|\psi_{l}^{d}\right|^{4}-2 J\left|\psi_{l}^{s}\right|\left|\psi_{l}^{d}\right| \cos \left(\theta^{d}-\theta^{s}\right)$

where the pseudo-order parameters $\psi_{l}^{s}$ and $\psi_{l}^{d}$ are nonzero and $\alpha_{s(d)}<0$. Note that two pseudo-order parameters are coupled by the inter-band Josephson coupling $J$, representing interactions between electrons in the $s$ - and $d$-bands. From Eq. (1), for $J>0$, it is clear that the free energy becomes a minimum for $\theta^{s}=$ $\theta^{d}$. However, for $J<0$, the free energy becomes a minimum for $\theta^{s}=\theta^{d}=\pi$. Thus, there is no phase frustration in two-gap superconductors for either $J>0$ or $J<0$.

The situation is different in the tunnel junction involving a two-gap superconductor and a one-gap superconductor. $\mathrm{Ng}$ and Nagaosa ( $\mathrm{Ng} \&$ Nagaosa 2009) suggested that the free energy density for a Josephson junction is given by

$$
\begin{aligned}
& F \cong \Theta(x)\left[-2 \tilde{J} \cos \left(\theta^{s}-\theta^{d}\right)+\widetilde{K}_{s}\left(\nabla \theta^{s}\right)^{2}+\widetilde{K}_{d}\left(\nabla \theta^{d}\right)^{2}\right]+ \\
& 2 \delta(x)\left[\widetilde{T}_{s} \cos \left(\theta^{s}-\theta\right)+\widetilde{T}_{d} \cos \left(\theta^{s}-\theta\right)\right]+ \\
& \Theta(-x) \widetilde{K}(\nabla \theta)^{2}+\widetilde{F},
\end{aligned}
$$

where $\quad \tilde{J}=J_{\text {in }}\left|\psi^{s}\right|\left|\psi^{d}\right|, \quad \tilde{T}_{s}=T_{s}|\psi|\left|\psi^{s}\right|, \quad \tilde{T}_{d}=$ $T_{d}|\psi|\left|\psi^{d}\right|, \widetilde{K}_{i}=K_{i}\left|\psi^{i}\right|^{2}, \tilde{F}$ is the part of free energy that is independent of phase angle, and the index $i=s, d$ denotes electronic bands in the two-gap superconductor.
Here, $T_{i}$ represents the coupling between the one-gap superconductor and the $i$-th electronic bands of the twogap superconductor. To study a deviation from the phase-locked state, one needs to minimize the free energy with respect to the phase variables. By minimizing the free energy with respect to the phase of the one-gap superconductor, one can obtain

$\widetilde{K} \nabla^{2} \theta=-\delta(x)\left(\tilde{T}_{s} \sin \theta^{s}-\widetilde{T}_{d} \sin \theta^{d}\right)$.

Here, the phase variable $\theta$ is set to zero (i.e., $\theta=0$ ) as a convenient reference point to measure the phases $\theta^{i}$. Note that Eq. (3) becomes $\widetilde{K} \nabla^{2} \theta=0$, away from the junction interface (i.e., $x \neq 0$ ). The solution of this equation may be written as $\theta=\beta_{s} x$.

Similarly, for the phases of the two pseudo-order parameters, one may obtain the equations of motion:

By matching the boundary condition to reflect the requirement that the current density is conserved at the junction interface, one can get

$\widetilde{K} \beta_{s}=-4 e\left[\widetilde{T}_{s} \sin \left(\theta^{0}+b_{d} \tan ^{-1} a\right)-\widetilde{T}_{d} \sin \left(\theta^{0}-\right.\right.$ $\left.\left.b_{s} \tan ^{-1} a\right)\right]$,

where $\left.b_{d}=4 \widetilde{K}_{d} /\left(\widetilde{K}_{s}+\widetilde{K}_{d}\right)\right)$ and $b_{s}=4 \widetilde{K}_{s} /\left(\widetilde{K}_{s}+\widetilde{K}_{d}\right)$.

In the ground state, the Josephson junction does not introduce any additional bulk energy to the system. This condition implies that there is no net current flow in the ground state of the system. Therefore, one can set $\beta_{s}=0$. Now Eq. (4) indicates that one can obtain

$$
\widetilde{T}_{s} \sin \theta^{0}-\widetilde{T}_{d} \sin \theta^{0}=0
$$

When $a=0$. This implies that $\theta^{0}=0$ or $\pi$. On the other hand, when $a \neq 0$, but $a$ is small, one may write $\partial F / \partial \theta^{s}=0$ as

$-\widetilde{T}_{d}\left[\sin \theta^{0} \cos \left(a b_{s}\right)-\cos \theta^{0} \sin \left(a b_{s}\right)\right]$.

Equation (5) indicates that $\theta^{0}$ must be different than 0 or $\pi$. If the phase difference between the two condensates becomes something other than 0 or $\pi$, then system is said to have phase frustration. The ground state of the junction with phase frustration has non-zero current flow, which breaks the time-reversal symmetry. For LJJ with two-gap and one-gap superconductor, the net current in the Josephson junction is zero (i.e., $J_{s}+J_{d}=0$ ) in the ground state (all these mathematical relations were derived). The time-reversal symmetry invariant state is represented by the trivial solutions of Eqs. (4) and (5). These solutions are $\theta^{0}=0$ or $\pi$ and $a=0$, indicating that $J_{s}=J_{d}=0$. Also, there are nontrivial solutions $\left( \pm \theta^{0}, \pm a\right) \neq 0$, representing the broken time-reversal states. These solutions are degenerate. The $\theta^{0} \lessgtr 0$ solutions correspond to two degenerate time- 
reversal pairs ( $\mathrm{Ng} \&$ Nagaosa 2009). In the BTRS state, the current loop circulates through the junction in momentum-space, and not in real space.

\section{Broken Time-Reversal Symmetry State in the Two- gap LJJ}

This is the main part of my research. The broken timereversal symmetry (BTRS) states in the Josephson junction with two-gap superconductors (as shown in fig. 1) are examined by using the free energy derived from the BCS Hamiltonian. By minimizing the free energy with respect to phase variables, one can determine the conditions for phase frustration, yielding the broken time-reversal symmetry state.

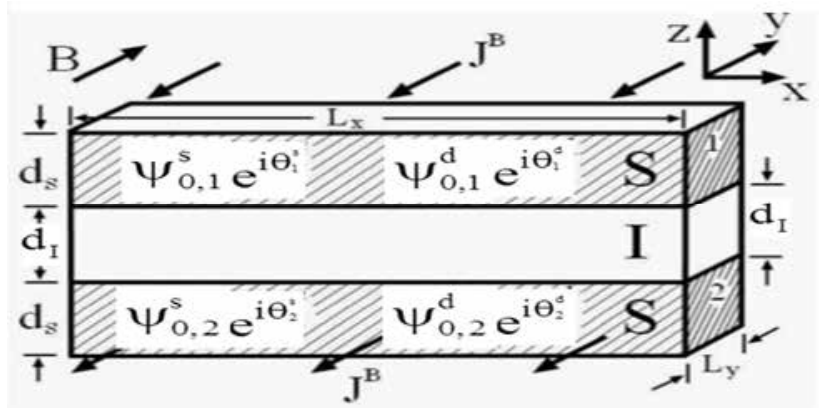

Fig. 1. A schematic diagram for a LJJ with two-gap superconductors is illustrated (Kim et al. 2012).

The BCS Hamiltonian extended for two-gap superconductors (Fetter \& Walecka 1971, Kim et al. 2012) is rewritten as $\widehat{H}=\widehat{H}_{T B, l}+\widehat{H}_{T}$. The Hamiltonians $\widehat{H}_{T B, l}$ and $\widehat{H}_{T}$ account for the contribution due to twogap superconductivity and electron tunneling between the two adjacent superconductor (S) layers. The twogap Hamiltonian $\widehat{H}_{T B, l}$ may be written in terms of Grassmann variables as

$\widehat{H}_{T B, l}=\int d r\left(\sum_{i=s, d} \varepsilon^{i} \bar{c}_{\sigma, l}^{i} c_{\sigma, l}^{i}+\widehat{H}_{l}^{\text {pair }}\right)$,

where $\varepsilon^{i}$ is the energy of electrons in the $i$-band $(i=s$, d) about the Fermi energy. The pairing interaction between electrons in the $l$-th $\mathrm{S}$ layer is given by

$\widehat{H}_{l}^{\text {pair }}=-V_{S S} \bar{c}_{\uparrow, l}^{S} \bar{c}_{\downarrow, l}^{S} c_{\downarrow, l}^{S} c_{\uparrow, l}^{S}-V_{d d} \bar{c}_{\uparrow, l}^{d} \bar{c}_{\downarrow, l}^{d} c_{\downarrow, l}^{d} c_{\uparrow, l}^{d}-$

$V_{s d}\left(\bar{c}_{\uparrow, l}^{S} \bar{c}_{\downarrow, l}^{S} c_{\downarrow, l}^{d} c_{\uparrow, l}^{d}+\right.$ h.c. $)$

Where $V_{i j}$ is the pairing interaction strength between electrons in the $i$ and $j$ bands and $\bar{c}_{\sigma, l}^{i}$ and $c_{\sigma, l}^{i}$ are the Grassmann variables. The Hamiltonian $\widehat{H}_{T}$ due to tunneling of an electron between the two adjacent $\mathrm{S}$ layers is given in terms of the tunneling matrix element $T_{i j}$ as

$\widehat{H}_{T}=\sum_{\sigma, i \neq j}\left(T_{i j} \bar{c}_{\sigma, 1}^{i} c_{\sigma, 2}^{j}+\right.$ h.c. $)$.
To obtain the free energy, one may start with the BCS Hamiltonian for a two-gap superconductor and carry out a number of steps. First, one may use the Nambu notation and Hubbard-Stratonovich transformation to simplify the partition function. Also, by using the Grassmann integrals to integrate the fermion fields, one can obtain the effective action for the system. I did a very long derivation to obtain effective action.

The free energy $F$ of the system may be obtained from the effective action as

$F=\frac{1}{\beta} S\left[\phi_{k}\right]$,

where $\phi_{k}$ is the Hubbard-Stratonovich field. Here the components of the auxiliary field

$\bar{\phi}_{k}=\left(\begin{array}{ll}\bar{\phi}_{k}^{s} & \bar{\phi}_{k}^{s}\end{array}\right)$ and $\phi_{k}=\left(\begin{array}{c}\phi_{k}^{s} \\ \phi_{k}^{d}\end{array}\right)$,

where $\phi_{k}^{i}=\Delta_{k}^{i} e^{i \theta^{i}}$. Using the effective action that was obtained in my derivation, one can write the free energy

$$
\begin{aligned}
& \beta F=\sum_{l}\left\{\int_{0}^{\beta} d \tau\left[\frac{\left|\Delta_{l}^{s}\right|^{2}}{g_{s s}}-\frac{\left|\Delta_{l}^{d}\right|^{2}}{g_{d d}}-\frac{2 g_{s d}}{g_{s s} g_{d d}} \Delta_{l}^{s} \Delta_{l}^{d} \cos \chi_{l}\right]\right. \\
& \left.+\int_{0}^{\beta} d \tau \sum_{i}\left[m_{i} \rho_{s}^{i} v_{s, l}^{i 2}+\frac{1}{8 \pi \mu_{i}^{2}}\left(\frac{\Phi_{0}}{2 \pi} \frac{\partial \theta_{l}^{i}}{\partial \tau}+\varphi_{l}\right)^{2}\right]\right\} \Theta( \pm z) \\
& +\left\{\int_{0}^{\beta} \frac{d \tau}{8 \pi}\left(\epsilon E_{z}^{2}+B_{y}^{2}\right)+\int_{0}^{\beta} \frac{d \tau}{d_{s}} \int_{0}^{\beta} d \tau^{\prime} B_{\alpha \beta}\right\} \delta(z)
\end{aligned}
$$

where $\chi_{l}=\theta_{l}^{d}-\theta_{l}^{s}$ and $B_{\alpha \beta}$ is the second order tunneling contribution to free energy. Also the phase difference between two $\mathrm{S}$ layers in the presence of an external magnetic field is

$\tilde{\phi}^{j i}=\theta_{2}^{j}-\theta_{1}^{i}-2 e A_{2,1}^{z}$,

and

$\vec{v}_{s, l}^{i}=\frac{1}{2 m_{i}}\left(\nabla \theta_{l}^{i}-\frac{2 \pi}{\Phi_{0}} \vec{A}_{l}\right)$,

is superfluid velocity. For simplicity, one can make the local approximation for the integral kernel $\tilde{\beta}^{j i}\left(\tau-\tau^{\prime}\right)$ in $B_{\alpha \beta}$ by writing $\tilde{\beta}^{j i}\left(\tau-\tau^{\prime}\right)=-\tilde{J}^{s S}\left(\tau-\tau^{\prime}\right) \delta\left(\tau-\tau^{\prime}\right)$.

Within this local approximation, noting that the Ohmic quasi-particle contributions $\tilde{\alpha}^{i j}\left(\tau-\tau^{\prime}\right) \cos \phi_{-}^{i j}\left(r, \tau ; r, \tau^{\prime}\right)$ in $B_{\alpha \beta}$ do not depend on the phases, one may write

$$
\begin{gathered}
\beta F=\sum_{l}\left\{\int_{0}^{\beta} d \tau\left[\frac{\left|\Delta_{l}^{s}\right|^{2}}{g_{s s}}-\frac{\left|\Delta_{l}^{d}\right|^{2}}{g_{d d}}-\frac{2 g_{s d}}{g_{s s} g_{d d}} \Delta_{l}^{s} \Delta_{l}^{d} \cos \chi_{l}\right]\right. \\
\left.+\int_{0}^{\beta} d \tau \sum_{i}\left[m_{i} \rho_{s}^{i} v_{s, l}^{i 2}+\frac{1}{8 \pi \mu_{i}^{2}}\left(\frac{\Phi_{0}}{2 \pi} \frac{\partial \theta_{l}^{i}}{\partial \tau}+\varphi_{l}\right)^{2}\right]\right\} \Theta( \pm z) \\
+\left\{\frac{1}{8 \pi} \int_{0}^{\beta} d \tau\left(\epsilon E_{z}^{2}+B_{y}^{2}\right)+\frac{1}{d_{s}} \int_{0}^{\beta} d \tau B_{\beta}\right\} \delta(z) .
\end{gathered}
$$


Note that, under local approximation, $B_{\alpha \beta}$ becomes

$B_{\alpha \beta}=B_{\beta}=J^{s s} \cos \tilde{\phi}^{s s}+J^{d d} \cos \tilde{\phi}^{d d}+J^{d s} \cos \tilde{\phi}^{d s}+$ $J^{s d} \cos \tilde{\phi}^{s d}$.

In the steady state, the free energy of the system in the absence of magnetic field is $F=\Theta( \pm z) \sum_{l}\left\{\frac{\left|\Delta_{l}^{s}\right|^{2}}{g_{s s}}-\right.$ $\frac{\left|\Delta_{l}^{d}\right|^{2}}{g_{d d}}-\frac{2 g_{s d}}{g_{s s} g_{d d}} \Delta_{l}^{s} \Delta_{l}^{d} \cos \chi_{l}$

$\left.+\frac{1}{4 m_{s}} \rho_{s}^{S}\left(\nabla \theta_{l}^{S}\right)^{2}+\frac{1}{4 m_{d}} \rho_{s}^{d}\left(\nabla \theta_{l}^{d}\right)^{2}\right\}-B_{\beta 0} \delta(z)$,

where

$B_{\beta 0}=J^{s s} \cos \phi^{s s}+J^{d d} \cos \phi^{d d}+J^{d s} \cos \left(\theta_{2}^{d}-\theta_{1}^{s}\right)+$

$J^{s d} \cos \left(\theta_{2}^{s}-\theta_{1}^{d}\right)$,

$\phi^{s s}=\theta_{2}^{s}-\theta_{1}^{s}$ and $\phi^{d d}=\theta_{2}^{d}-\theta_{1}^{d}$.

Now the phase configuration that minimizes the free energy is discussed. In the steady state, the phase part of the free energy $F_{\theta}=F-F_{0}$ of the LJJ based on twogap superconductors is

$F_{\theta}=-\bar{g}_{s d}\left(\cos \chi_{1}+\cos \chi_{2}\right)-\bar{J}^{s s}\left(\cos \phi^{s s}+\right.$

$\left.\cos \phi^{d d}\right)-\bar{J}^{s d}\left[\cos \left(\theta_{2}^{s}-\theta_{1}^{d}\right)+\cos \left(\theta_{2}^{d}-\theta_{1}^{s}\right)\right]$,

where $\bar{g}_{s d}=2 g_{s d} \Delta_{l}^{s} \Delta_{l}^{d} / g_{s s} g_{s d}, \quad \chi_{1}=\theta_{1}^{d}-\theta_{1}^{s}$, and $\chi_{2}=\theta_{2}^{d}-\theta_{2}^{s}$. Note that one may set that $\bar{J}^{s s}=$ $J^{s s} / d_{s}=J^{d d} / d_{s}, \quad$ and $\bar{J}^{s d}=J^{s d} / d_{s}=J^{d s} / d_{s}$ for simplicity. Here $F_{0}$ is the part of the free energy density which is independent of the $\theta$ 's. Note that $g_{s s}=$ $\operatorname{det}(V) / V^{d d}, \quad g_{d d}=\operatorname{det}(V) / V^{s s}, \quad$ and $\quad g_{s d}=$ $\operatorname{det}(V) V^{s d} / V^{d d} V^{s s}$, where $V$ is given in Eq. (8). To examine the phase configurations which minimize the free energy density, the first derivative of $F_{\theta}$ with respect to phase variables is set to zero. The extrema of the free energy function $F\left(\theta_{1}^{s}, \theta_{1}^{d}, \theta_{2}^{s}, \theta_{2}^{d}\right)=$ $F\left(\chi_{1}, \chi_{2}, \phi^{s s}, \phi^{d d}\right)$ may be found by using the two sets of conditions. The first set of conditions is $\phi^{d d}=$ $\phi^{s s}+\chi_{2}-\chi_{1}, \quad \phi^{s s}+\frac{\chi_{2}-\chi_{1}}{2}=n \pi, \quad$ and $\bar{g}_{s d} \sin \left(\frac{\chi_{2}+\chi_{1}}{2}-\frac{\chi_{2}-\chi_{1}}{2}\right)+(-1)^{n}\left(-\bar{J}^{s s} \sin \frac{\chi_{2}-\chi_{1}}{2}+\right.$ $\left.\bar{J}^{s d} \sin \frac{\chi_{2}+\chi_{1}}{2}\right)=0$.

The second set of conditions is $\phi^{d d}=\phi^{s s}+\chi_{2}-$ $\chi_{1}, J^{s s} \cos \left(\frac{\chi_{2}-\chi_{1}}{2}\right)+J^{s d} \cos \left(\frac{\chi_{2}+\chi_{1}}{2}\right)=0$ and

$$
\begin{aligned}
\bar{g}_{s d} \sin \left(\frac{\chi_{2}+\chi_{1}}{2}-\frac{\chi_{2}-\chi_{1}}{2}\right) & \\
& -\cos \left(\phi^{s s}\right. \\
& \left.+\frac{\chi_{2}-\chi_{1}}{2}\right)\left(\bar{J}^{s s} \sin \frac{\chi_{2}-\chi_{1}}{2}-\bar{J}^{s d} \sin \frac{\chi_{2}+\chi_{1}}{2}\right) \\
& =0 .
\end{aligned}
$$

Using the conditions for minimum free energy, above equation is solved for $\mathrm{z}=0$ numerically. To study the phase frustration in the ground state of the LJJ, the free energy $F_{\theta} / J^{S S}$ is plotted in the Fig. 2 as a function of inter-band relative phase for (a) $g_{S D}=\bar{g}_{s d} / J^{S S}=1.0$, $J_{S D}=\bar{J}^{s d} / J^{S S}=1.0, \phi^{S S}=0.0$ (b) $J_{S D}=1.0 g_{S D}=-1$, $\phi^{S S}=0.0$ and (c) $g_{S D}=-1.0, J_{S D}=1.0, \phi^{S S}=1.4$. Also, in Fig. 3, the free energy contours are plotted as a function of relative phase for the same set of parameters as used in Fig. 2. These free energy plots indicate that the value of relative phases $\left(\chi_{1}^{o}, \chi_{2}^{o}\right)$ for the free energy minimum depends on $\phi^{s s}$. From the free energy contour plot of Fig. 3, one can easily see that the ground state value for $\left(\chi_{1}^{o}, \chi_{2}^{o}\right)$ is $(0,0)$, when the phase difference $\phi^{s S}$ across the two adjacent layers is zero (i.e., $\phi^{s S}=0$ ). However, when $\phi^{S S} \neq 0$, the free energy minimum occurs for $g_{S D}=-1.0$ and $J_{S D}=1.0$ at a non-zero value of $\left(\chi_{1}^{o}, \chi_{2}^{o}\right)$, indicating the appearance of phase frustration in the ground state. This dependence on $\phi^{s S}$ may be seen easily in Fig. 2c. Similarly for $g_{S D}=-1.0, J_{S D}=1.0$, and $\phi^{S S}=0.0$, the free energy surface and contours in the $\left(\chi_{1}, \chi_{2}\right)$ space is shown in Figs. $2 b$ and $3 b$, respectively.

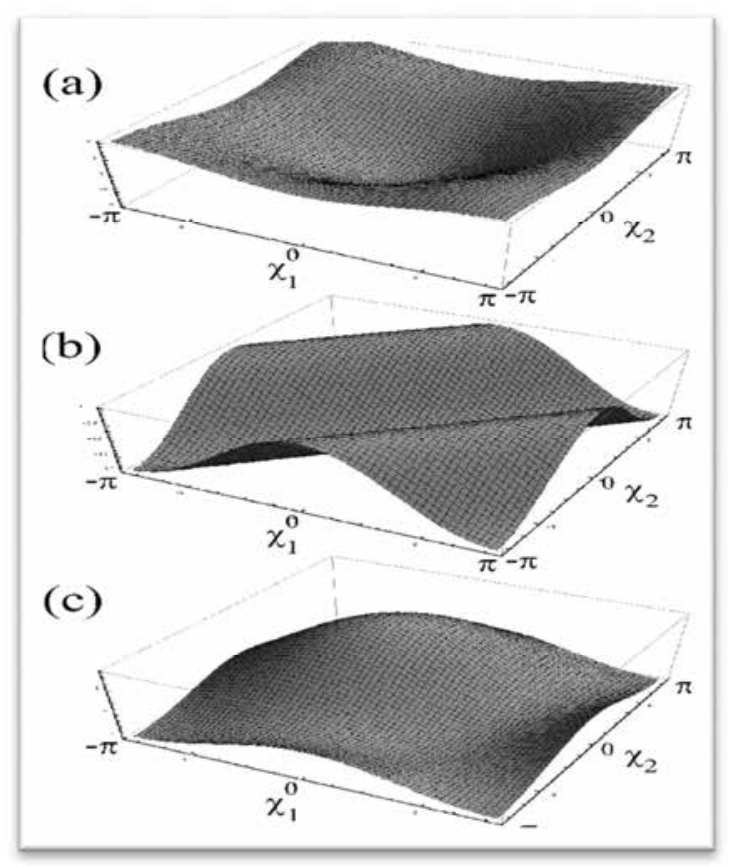

Fig. 2. The free energy $F_{\theta} / J^{s s}$ is plotted as a function of inter-band relative phase difference $\chi_{1}$ and $\chi_{2}$ for (a) $g_{S D}=\bar{g}_{s d} / J^{s s}=1.0, J_{S D}=\bar{J}^{s d} / J^{s s}=1.0, \phi^{s s}=0$ (b) $J_{S D}=1.0, g_{S D}=-1, \phi^{s S}=0$ and (c) $g_{S D}=-1.0$, $J_{S D}=1.0, \phi^{s S}=1.4$. These free energy surfaces illustrate the dependence of the ground state phase configuration on the parameters $g_{S D}, J_{S D}$, and $\phi^{s s}$. 


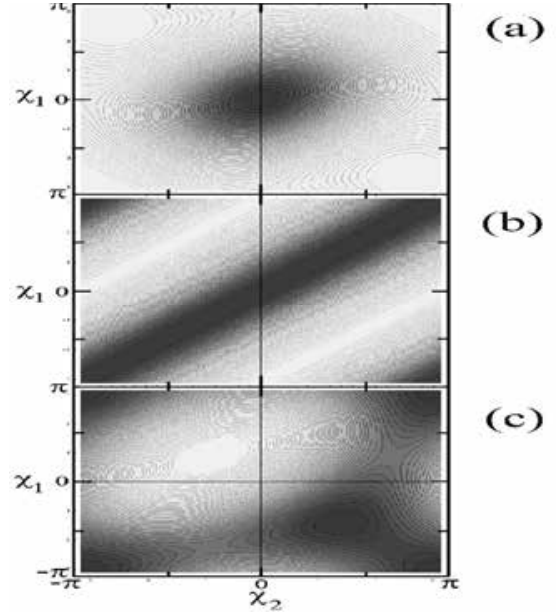

Fig. 3. The free energy $F_{\theta} / J^{s s}$ is plotted as a function of inter-band relative phase difference $\chi_{1}$ and $\chi_{2}$ for (a) $g_{S D}=\bar{g}_{s d} / J^{s s}=1.0, J_{S D}=\bar{J}^{s d} / J^{s s}=1.0, \phi^{s s}=0$ (b) $J_{S D}=1.0, g_{S D}=-1, \phi^{s s}=0$ and (c) $g_{S D}=-1.0$, $J_{S D}=1.0, \phi^{s s}=1.4$. These free energy contours illustrate the location of the minimum free energy and to estimate the coordinates $\left(\chi_{1}, \chi_{2}\right)$ for given parameters $g_{S D}$, $J_{S D}$, and $\phi^{s s}$.

One may estimate the phase frustration in the ground state from the phase equation of motion derived from the free energy of Eq. (17). One uses the Euler-Lagrange equations for different phase variables to obtain the equations of motion. For an example, for the $\theta_{2}^{d}$ variable, the equation of motion is given by

$$
\begin{aligned}
& \frac{\hbar^{2} \rho_{s}^{d}}{2 m_{d}} \nabla^{2} \theta_{2}^{d}-\frac{2 g_{s d}}{g_{s s} g_{d d}} \Delta_{2}^{s} \Delta_{2}^{d} \sin \left(\theta_{2}^{d}-\theta_{2}^{s}\right) \\
& =\left[\frac{J^{d d}}{d_{s}} \sin \left(\theta_{2}^{d}-\theta_{1}^{d}\right)+\frac{J^{d s}}{d_{s}} \sin \left(\theta_{2}^{d}-\theta_{1}^{s}\right)\right] \delta(z) .
\end{aligned}
$$

Similar equations of motion can be obtained for the phase variables $\theta_{2}^{s}, \theta_{1}^{d}$, and $\theta_{1}^{s}$. When the EulerLagrange equations for $\theta_{2}^{d}$ and $\theta_{2}^{s}$ are added, one can obtain

$\nabla^{2}\left(\theta_{2}^{d}-\theta_{2}^{s}\right)-\frac{g_{s d}}{g_{s s} g_{d d}} \Delta_{2}^{s} \Delta_{2}^{d} \frac{4}{\hbar^{2}}\left(\frac{m_{d}}{\rho_{s}^{d}}+\frac{m_{s}}{\rho_{s}^{s}}\right) \sin \left(\theta_{2}^{d}-\right.$

$\left.\theta_{2}^{s}\right)=0$

for $z>0$. Noting that $\chi_{2}=\theta_{2}^{s}-\theta_{2}^{d}$, one can write

$\nabla^{2} \chi_{2}-\frac{1}{\lambda^{2}} \sin \chi_{2}=0$,

where

$\frac{1}{\lambda^{2}}=\frac{g_{s d}}{g_{s s} g_{d d}} \Delta_{2}^{s} \Delta_{2}^{d} 4\left(\frac{m_{d}}{\rho_{s}^{d}}+\frac{m_{s}}{\rho_{s}^{s}}\right)$.

A single-soliton solution to the sine-Gordon equation of Eq. (20) for the relative phase $\chi_{2}$ is given by

$\chi_{2}(z)=4 \tan ^{-1}\left(a_{2} e^{-\frac{z}{\lambda_{2}}}\right)$.
One can decompose Eq. (21) and obtain the expression for $\theta_{2}^{d}$ and $\theta_{2}^{s}$ as

$\theta_{2}^{d}=\beta_{2}^{0} z+\theta_{2}^{0}+\pi-\frac{4 \rho_{s}^{s} m_{d}}{\rho_{s}^{d} m_{s}+\rho_{s}^{s} m_{d}} \tan ^{-1}\left(a_{2} e^{-\frac{z}{\lambda_{2}}}\right)$

and

$\theta_{2}^{s}=\beta_{2}^{0} z+\theta_{2}^{0}+\frac{4 \rho_{s}^{d} m_{s}}{\rho_{s}^{d} m_{s}+\rho_{s}^{s} m_{d}} \tan ^{-1}\left(a_{2} e^{-\frac{z}{\lambda_{2}}}\right)$,

respectively. Similarly, one may add the Euler-Lagrange equation for $\theta_{1}^{d}$ and $\theta_{1}^{s}$ to obtain the sine-Gordon equation for $\chi_{1}=\theta_{1}^{s}-\theta_{1}^{d} \quad$ (i.e., for $z<0$ ). By following the decomposition approach for $\chi_{2}$, one can write the phase variables $\theta_{1}^{d}$ and $\theta_{1}^{s}$ as

$\theta_{1}^{d}=\beta_{1}^{0} z+\theta_{1}^{0}+\pi-\frac{4 \rho_{s}^{s} m_{d}}{\rho_{s}^{d} m_{s}+\rho_{s}^{s} m_{d}} \tan ^{-1}\left(a_{1} e^{-\frac{z}{\lambda_{1}}}\right)$,

and

$\theta_{1}^{s}=\beta_{1}^{0} z+\theta_{1}^{0}+\frac{4 \rho_{s}^{d} m_{s}}{\rho_{s}^{d} m_{s}+\rho_{s}^{s} m_{d}} \tan ^{-1}\left(a_{1} e^{-\frac{z}{\lambda_{1}}}\right)$,

respectively. The current density $\tilde{J}_{l}^{i}=2 e\left(\partial F / \partial \theta_{l}^{i}\right)$ for $l=1,2$ at $z=0$ leads to

$\tilde{J}_{2}^{s}=\frac{2 e}{\hbar}\left[\frac{J^{s s}}{d_{s}} \sin \left(\theta_{2}^{s}-\theta_{1}^{s}\right)+\frac{J^{s d}}{d_{s}} \sin \left(\theta_{2}^{s}-\theta_{1}^{d}\right)\right]$,

and

$\tilde{J}_{1}^{s}=\frac{2 e}{\hbar}\left[-\frac{J^{s s}}{d_{s}} \sin \left(\theta_{2}^{s}-\theta_{1}^{s}\right)-\frac{J^{s d}}{d_{s}} \sin \left(\theta_{2}^{d}-\theta_{1}^{s}\right)\right]$,

Similar relation for $\tilde{J}_{1}^{d}$ and $\tilde{J}_{1}^{s}$ can be obtained easily. Also, noting that the current density is given by

$\vec{J} \cong \frac{1}{\Lambda} \frac{\Phi_{0}}{2 \pi} \nabla \theta=2 e \frac{d F}{d \theta}$,

one may write for $\tilde{J}_{1}^{s}=2 e \frac{\hbar \rho_{s}^{s}}{m_{s}} \beta_{1}^{0}+\frac{b_{1} a_{1}}{\lambda_{1}\left(1+a_{1}\right)}$

$=-\frac{J^{s s}}{d_{s}} \sin \left[\delta \theta^{o}+b_{2}\left(\tan ^{-1} a_{2}+\tan ^{-1} a_{1}\right)\right]$

$-\frac{J^{s d}}{d_{s}} \sin \left[\delta \theta^{o}+\pi+b_{2}\left(\tan ^{-1} a_{2}+\tan ^{-1} a_{1}\right)\right]$,

where $\delta \theta^{o}=\theta_{2}^{o}-\theta_{1}^{o}$ denotes the relative phase constant which does not depend on position, $b_{1}=$ $8 e \rho_{s}^{s} \rho_{s}^{d} /\left(\rho_{s}^{d} m_{s}+\rho_{s}^{s} m_{d}\right) \quad$ and $b_{2}=4 \rho_{s}^{d} m_{s} /\left(\rho_{s}^{d} m_{s}+\rho_{s}^{s} m_{d}\right)$. For the simple case of $a_{1}=0$ and $a_{2}=0$, one can obtain

$\frac{2 e \rho_{s}^{s}}{m_{s}} \beta_{1}^{0}=-\frac{J^{s s}}{d_{s}} \sin \left(\delta \theta^{o}\right)+\frac{J^{d s}}{d_{s}} \sin \left(\delta \theta^{o}\right)$.

This means that when $\beta_{1}^{0}=0$ and $\theta_{2}^{0}=(0, \pi)=\theta_{1}^{0}$, the ground state is time-reversal symmetry invariant since $\tilde{J}_{1}^{s}=0$ and $\tilde{J}_{1}^{d}=0$. On the other hand, when $a_{1} \neq 0$ and $a_{2} \neq 0$, but both $a_{1}$ and $a_{2}$ are small, one may expand $\tan ^{-1} a=a-\frac{a^{3}}{3}+\frac{a^{5}}{5}+\cdots$.

and write Eq. (29) as $\frac{2 e \rho_{s}}{m_{s}} \beta_{1}^{0}+\frac{b_{1} a_{1}}{\lambda_{1}} \cong$ 
$-\frac{J^{s s}}{d_{s}} \sin \left[\delta \theta^{o}+b_{2}\left(a_{2}+a_{1}\right)\right]+\frac{J^{d s}}{d_{s}} \sin \left(\delta \theta^{o}+b_{3} a_{2}+b_{2} a_{1}\right),(30)$

indicating the relations between the constants $\beta_{1}^{0}, \delta \theta^{\circ}$, $a_{1}$, and $a_{2}$.

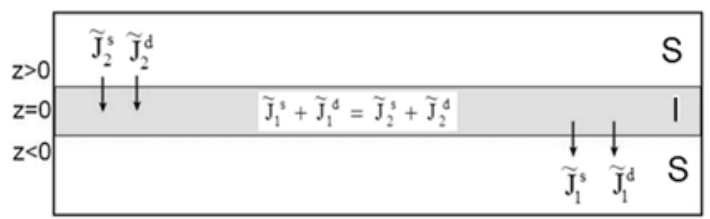

Fig. 4. Boundary conditions for current density in the ground state of LJJ are schematically illustrated. A LJJ with two layers of two-gap superconductors which are separated by an insulator in the z-direction is shown. The $l=2$ and $l=1$ superconductor layers are above $(z>0)$ and below $(z<0)$ the junction interface.

At the junction interface (i.e., $\mathrm{z}=0$ ), the current density is conserved (Fig. 4). This boundary condition for the current densities may be summarized as

$\tilde{J}_{1}^{s}+\tilde{J}_{1}^{d}=\left(\tilde{J}_{2}^{s}+\tilde{J}_{2}^{d}\right)$.

One may see how this boundary condition may lead to phase frustration by first evaluating the current densities at $z=0$ as

$\tilde{J}_{2}^{s}=2 e \frac{\partial F}{\partial \theta_{2}^{s}}=2 e\left[\frac{J^{s s}}{d_{s}} \sin \left(\theta_{2}^{s}-\theta_{1}^{s}\right)+\frac{J^{s d}}{d_{s}} \sin \left(\theta_{2}^{s}-\theta_{1}^{d}\right)\right]$,

$\tilde{J}_{2}^{d}=2 e \frac{\partial F}{\partial \theta_{2}^{d}}=2 e\left[\frac{J^{d d}}{d_{s}} \sin \left(\theta_{2}^{d}-\theta_{1}^{d}\right)+\frac{J^{d s}}{d_{s}} \sin \left(\theta_{2}^{d}-\theta_{1}^{s}\right)\right]$,

$\tilde{J}_{1}^{s}=2 e \frac{\partial F}{\partial \theta_{1}^{s}}=-2 e\left[\frac{J^{s s}}{d_{s}} \sin \left(\theta_{2}^{s}-\theta_{1}^{s}\right)+\frac{J^{d s}}{d_{s}} \sin \left(\theta_{2}^{d}-\theta_{1}^{s}\right)\right]$,

and

$\tilde{J}_{1}^{d}=2 e \frac{\partial F}{\partial \theta_{1}^{d}}=-2 e\left[\frac{J^{d d}}{d_{s}} \sin \left(\theta_{2}^{d}-\theta_{1}^{d}\right)+\frac{J^{s d}}{d_{s}} \sin \left(\theta_{2}^{s}-\theta_{1}^{d}\right)\right]$.

Now, one can impose the boundary condition of Eq. (34) at $z=0$. Applying the boundary condition, one can show that $\tilde{J}_{1}^{s}=0$ when $\beta_{1}^{0}=0, a_{1}=a_{2}=0$ and $\theta_{1}^{0}=(0, \pi)=\theta_{2}^{0}$. This means that $\tilde{J}_{1}^{s}=\tilde{J}_{1}^{d}=\tilde{J}_{2}^{s}=$ $\tilde{J}_{2}^{d}=0$, indicating that there is no net current flowing through the system in the ground state. This solution obeys the time-reversal symmetry. Another solution that satisfies the boundary condition at $\mathrm{z}=0$ may also be found. The solution $\tilde{J}_{1}^{s}+\tilde{J}_{1}^{d}=0$ (and $\tilde{J}_{2}^{s}+\tilde{J}_{2}^{d}=0$ ) indicates that the net current density is zero when $\tilde{J}_{1}^{s}=$ $-\tilde{J}_{1}^{d}$ (and $\tilde{J}_{2}^{s}=-\tilde{J}_{2}^{d}$ ). This solution breaks time-reversal symmetry. The appearance of the BTRS state in the ground state is indicated by the non-zero value of the relative phase constant $\delta \theta^{\circ}=\theta_{2}^{0}-\theta_{1}^{0}$. This constant may be computed by evaluating the phases at the junction interface (i.e., $z=0$ ). At $z=0$, the phases can be obtained using Eqs. (27) and (28) as

$\theta_{l}^{d}=\theta_{l}^{0}+\pi-\frac{4 \rho_{s}^{s} m_{d}}{\rho_{s}^{d} m_{s}+\rho_{s}^{s} m_{d}} \tan ^{-1} a_{l}$ and

$\theta_{l}^{s}=\theta_{l}^{0}+\frac{4 \rho_{s}^{s} m_{d}}{\rho_{s}^{d} m_{s}+\rho_{s}^{s} m_{d}} \tan ^{-1} a_{l}$

Subtracting Eq. (36) from Eq. (37), one can obtain

$\chi_{l}=\pi-4 \tan ^{-1} a_{l}$.

Now, the relative phase constant $\delta \theta^{\circ}=\theta_{2}^{0}-\theta_{1}^{0}$ indicating that the ground state breaks the time-reversal symmetry is obtained by imposing the boundary conditions $\tilde{J}_{1}^{s}=-\tilde{J}_{1}^{d}$ and $\tilde{J}_{2}^{s}=-\tilde{J}_{2}^{d}$. By using Eqs. (36) and (37), one can obtain

$\delta \theta^{\circ}=$

$\tan ^{-1}\left\{\frac{\sin K_{d}\left(\chi_{2}^{o}-\chi_{1}^{o}\right)-\sin K_{s}\left(\chi_{\chi^{o}}^{o}-\chi_{1}^{o}\right)-\frac{J^{s d}}{J^{s}\left[\sin \left(K_{s} \chi_{2}^{o}-K_{d} \chi_{1}^{o}\right)-\sin \left(K_{d} \chi_{2}^{o}-K_{s} \chi_{1}^{o}\right)\right]}}{\cos K_{d}\left(\chi_{2}^{o}-\chi_{1}^{o}\right)+\cos K_{s}\left(\chi_{2}^{o}-\chi_{1}^{o}\right)+\frac{J^{s}}{J^{s s}}\left[\cos \left(K_{s} \chi_{2}^{o}-K_{d} \chi_{1}^{o}\right)+\cos \left(K_{d} \chi_{2}^{o}-K_{s} \chi_{1}^{o}\right)\right]}\right\}$

where $\chi_{l}^{o}$ is the relative phase of the two condensates of $l$-th $\mathrm{S}$ layer in the ground state where the system has minimum free energy. From Eq. (34) it is clear that $\theta_{2}^{o}-\theta_{1}^{o}=0$ when the relative phases for both the $\mathrm{S}$ layers are zero. Similarly, when two relative phases are equal, but have opposite signs (i.e. $\chi_{2}^{o}=\chi^{o}$ and $\chi_{1}^{o}=$ $-\chi^{o}$ ), one can obtain

$\delta \theta^{o}=\tan ^{-1}\left[\frac{\sin 2 K_{S} \chi^{o}-\sin 2 K_{d} \chi^{o}-\frac{2 J^{s d}}{J^{s S}} \sin \chi^{o}\left(K_{d}-K_{S}\right)}{\cos 2 K_{s} \chi^{o}+\cos 2 K_{d} \chi^{o}+\frac{2 J^{s d}}{J^{S S}} \cos \chi^{0}\left(K_{d}-K_{S}\right)}\right]$.

To study the variation of phase constant $\delta \theta^{\circ}=\theta_{2}^{0}-\theta_{1}^{0}$ as a function of $\phi^{S S}$, the relative phases at the minimum free energy was obtained by plotting free energy as a function of relative phase as shown in Fig. 5. For a given value of $\phi^{s S}$, the phase configuration $\left(\chi_{1}, \chi_{2}\right)$ which corresponds to the ground state $\left(\chi_{1}^{o}, \chi_{2}^{o}\right)$ is determined. This value is substituted into Eq. (40) to calculate $\delta \theta^{\circ}$ numerically. In Fig. 5, a plot of $\delta \theta^{\circ}$ versus $\phi^{s S}$ is shown to illustrate the dependence on $K_{S}$. The three lines corresponds to $K_{S}=0.49$ (solid line), $K_{s}=0.47$ (dashed line), and $K_{S}=0.45$ (dotdashed line). The curves in Fig. 5 show that the relative phase constant $\delta \theta^{\circ}$ in the ground state varies linearly with $\phi^{S S}$. Also, one can see from Fig. 6 that the rate of variation $\delta \theta^{\circ}$ of for $K_{s}=0.45$ is higher than that for $K_{s}=0.47$ and $K_{s}=0.49$. This dependence on $K_{s}$ indicates that greater charge imbalance between the charge densities of the $\mathrm{s}$ and d-band present in the system leads to stronger frustration in the ground state phase configurations. 


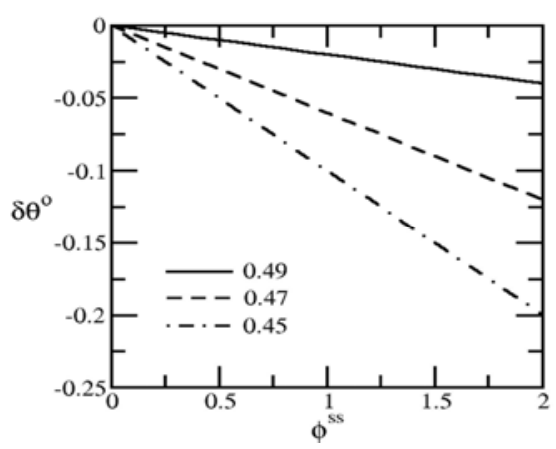

Fig. 5. The phase constant $\delta \theta^{\circ}$ for the ground state is plotted as a function of $\phi^{s s}$ for three different values of $K_{s}=0.49$ (solid line), 0.47 (dashed line), and 0.45 (dotdashed line). These curves illustrate the effect of relative phase on the phase constant.

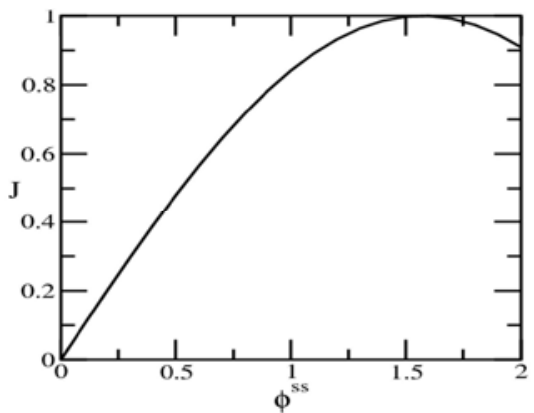

Fig. 6. The current density $J$ in the ground state is plotted as a function of $\phi^{s s}$. The curve illustrates the effect of relative phase on the current density at the junction interface $(z=0)$.

In Fig. 6 the current density $J=\left|J_{1}^{S}\right|$ at the junction interface (i.e., $\mathrm{z}=0$ ) is plotted as a function of the phase difference $\phi^{s s}$. The curve indicates clearly that current density varies as the sine function with the phase difference. The results of phase frustration in the ground state of the Josephson junction with two-gap superconductors and its consequences are discussed in the next section.

\section{RESULTS AND DISCUSSION}

To understand the time-reversal symmetry invariant (TRSI) and broken time-reversal symmetry (BTRS) state in a Josephson junction with two-gap superconductors, I investigated the ground state phase configuration. The ground state phase configuration was obtained by minimizing the free energy of the LJJ with respect to phase variables in the absence of an external magnetic field. The boundary conditions were obtained at the junction interface. Applying the boundary conditions, the conditions for TRSI and BTRS state were obtained. When the current density in the $s$ and $d$ electronic bands in the ground state are zero, the ground state corresponds to TRSI state. In this case, the relative phase constants attain the values either 0 or $\pi$. Although the net current density in the ground state is zero, the individual currents can be non-zero. In this case, $\tilde{J}_{l}^{s}=-\tilde{J}_{l}^{d}$ and the relative phase constants differsfrom 0 or $\pi$. This solution breaks time-reversal symmetry. The relative phase in the ground state was numerically calculated. The phase configuration of the ground state was determined from the free energy calculation as a functions of relative phases $\left(\chi_{1}, \chi_{2}\right)$ for different values of inter-band current and Josephson current. When the ground state corresponds to the BTRS, the relative phase constant $\delta \theta^{\circ}$ computed as a function of phase difference $\phi^{s S}$ showed linearly dependence. The variation of current density $\left.U_{1}^{s}\right\rfloor$ with the phase difference $\phi^{s s}$ behaves as the sine-curve.

\section{REFERENCES}

Ng, T.K. and Nagaosa N. 2009. Broken time-reversal symmetry in the Josephson junction involving twoband superconductors. EPL 87: 17003.

Lee, W.-C., Zhank, S. C., Wu, C. 2009. Pairing state with a time-reversal symmetry breaking in FeAs-based superconductors. PRL102: $217002 .$.

Tanaka, Y., Shirage P.M., Iyo A. 2010. Time-reversal symmetry-breaking in two-band superconductors. Physica C 470: 2023-2026.

Tanaka, Y., Iyo A., Tokiwa, K., Watanabe T., Crisan A., Sundaresan A., Terada, N. 2010. Topologicalstructure of the inter-band phase difference soliton in two-band superconductors. Physica C 470 : 2010-2012.

Lin, S.Z., Hu, X. 2011. Topological Excitations in multi-band superconductivity arXive: 1111.3850 vl [cond-mat. Supr-con] 16 Nov.

Chung, S.G. 2002. Time reversal symmetry based on two-gap superconductors. Physica E 12: 931.

Bardeen, J., Cooper, L.N., Schrieffer, J.R. 1957. Theory of Superconductivity. Phys. Rev. 108: 1175.

Loufwander, T., Shumeiko V. S., Wendin G. 2000. Timereversal symmetry braking at Garaud, J., Carlstroum, J., Babaev, E. 2011. Topological solutions in threeband superconductors with broken time reversalsymmetry. Phys. Rev. Lett. 107: 197001.

Hu, X., Wang, Z. 2011. Time reversal broken superconductivity induced by frustrated intercomponent couplings, e-print arXiv: 1103.0123 [Cons-mat. Supr-con].

Platt, C., Thomale, R., Honerkamp, C., Zhang, S. C., Hanke, W. 2011. Mechanism for a pairing state with time reversal symmetry breaking in Iron-based superconductors.e-printarXive: 11006.5964 [Cons-mat. Supr-con].

Fetter, A.L., Walecka, J.D. 1971. Quantum theory of Many Particle (McGraw Hill, NewYork, pp-225.

Kim, J.H., Ghimire, B.R., Tsai, H.Y. 2012. Fluxon dynamics in LJJ with two-gap Superconductors. Phys. Rev. B85: 134511. 